\title{
Gastrointestinal stromal tumors: associations between contrast- enhanced CT images and KIT exon 11 gene mutation
}

\author{
Xijiao Liu ${ }^{1 \#}$, Yuan Yin ${ }^{2 \#}$, Xiaozhou Wang ${ }^{3 \#}$, Caiwei Yang ${ }^{1}$, Shang Wan ${ }^{1}$, Xiaonan Yin ${ }^{2}$, Tingfan Wu ${ }^{4}$, \\ Huijiao Chen ${ }^{5}$, Zhongming $\mathrm{Xu}^{3}$, Xin $\mathrm{Li}^{6}$, Bin Song ${ }^{1}$, Bo Zhang ${ }^{2}$ \\ ${ }^{1}$ Department of Radiology, West China Hospital, Sichuan University, Chengdu, China; ${ }^{2}$ Department of Gastrointestinal Surgery, West China \\ Hospital, Sichuan University, Chengdu, China; ${ }^{3}$ Department of Radiology, Meishan People's Hospital, Meishan, China; ${ }^{4}$ Clinical Education Team, \\ GE Healthcare China, Shanghai, China; ${ }^{5}$ Department of Pathology, West China Hospital, Sichuan University, Chengdu, China; ${ }^{6}$ Global Research, \\ GE Healthcare China, Shanghai, China \\ Contributions: (I) Conception and design: B Song, B Zhang; (II) Administrative support: B Song, B Zhang, H Chen, X Li; (III) Provision of study \\ materials or patients: B Song, B Zhang, H Chen; (IV) Collection and assembly of data: X Liu, Y Yin, X Wang, C Yang, S Wan, X Yin, F Ting, H \\ Chen, Z Xu, X Li; (IV) Data analysis and interpretation: X Liu, Y Yin, X Wang, C Yang, S Wan, X Yin, F Wu, H Chen, Z Xu, X Li; (VI) Manuscript \\ writing: All authors; (VII) Final approval of manuscript: All authors. \\ "These authors contributed equally to this work. \\ Correspondence to: Prof. Bin Song. Department of Radiology, West China Hospital, Sichuan University, Chengdu 610041, China. \\ Email: songlab_radiology@163.com; Prof. Bo Zhang. Department of Gastrointestinal Surgery, West China Hospital, Sichuan University, Chengdu \\ 610041, China. Email: hxwcwk@126.com.
}

Background: Mutation screening for gastrointestinal stromal tumor (GIST) is crucial and the c kit gene (KIT) exon 11 mutation is the most common type. This study aimed to explore the associations between GIST with KIT exon 11 mutation and contrast-enhanced computed tomography (CT) images.

Methods: Pathologically proven GISTs with definitive genotype testing results in our hospital were retrospectively included. Abdominal contrast-enhanced CT images were analyzed. Conventional CT image features and radiomic features were recorded and extracted to build the following models: model ${ }_{[\mathrm{CT}]}$, model ${ }_{\text {[radiomic + clinic] }}$ and model ${ }_{[\mathrm{CT}+\text { radiomic + clinic] }}$. The diagnostic performances of GISTs with KIT exon 11 mutation and KIT exon 11 deletion involving codons 557-558 were evaluated.

Results: In total, 327 GISTs (255 with KIT exon 11 mutation, and 73 with KIT exon 11 mutation deletion involving codons 557-558) were included. Significant CT features were found for GISTs with KIT exon 11 mutation. The area under curves (AUCs) of the models for KIT exon 11 mutation were 0.7158, 0.7530, and 0.8375 in the training cohort, and $0.6777,0.7349$, and 0.8105 in validation cohort, respectively. The AUCs of the models for KIT exon 11 mutation deletion involving codons 557-558 were 0.7155, 8621, and 0.8691 in the training cohort, and $0.7099,0.8355$, and 0.8488 in the validation cohort, respectively. The model $[$ CT + radiomic + clinic] demonstrated the highest AUCs for prediction of KIT exon 11 mutation and those with deletion involving codons 557-558 $(\mathrm{P}<0.05)$, respectively. The model ${ }_{\text {[radiomic }+ \text { clinic] }}$ showed higher diagnostic performance than model ${ }_{[\mathrm{CT}]}$ significantly.

Conclusions: Our results demonstrated the associations between GIST with KIT exon 11 mutation and contrast-enhanced CT images. We found combing conventional image analysis and texture analysis is a useful tool to distinguish GIST with KIT exon 11 mutation. CT radiogenomics exhibited good application potential in predict the KIT exon 11 mutation of GIST.

Keywords: Gastrointestinal stromal tumor (GIST); mutation; KIT exon 11; computed tomography; imaging genomics

Submitted Jul 08, 2021. Accepted for publication Sep 02, 2021.

doi: 10.21037/atm-21-3811

View this article at: https://dx.doi.org/10.21037/atm-21-3811 


\section{Introduction}

Gastrointestinal stromal tumor (GIST) is the most common mesenchymal tumor of the gastrointestinal tract, with an incidence of up to $7-15$ cases per million $(1,2)$. It usually affects adults aged between 50 and 70 years without clear sex predilection, and can occur in any site of the gastrointestinal system. The biological behavior of GISTs varies from benign to malignant, and curative surgical resection is the first-line option for treatment. Small molecule tyrosine kinase inhibitors (TKIs), such as imatinib, significantly improve the prognosis of GISTs.

Most GISTs exhibit an activated mutation in the gene encoding c-kit gene (KIT) or platelet-derived growth factor receptor alpha (PDGFRA) receptor tyrosine kinase (3). Approximately $95 \%$ of GISTs arising in adults over-express the KIT protein and $80 \%$ of GISTs involve KIT gene mutations. More than $70 \%$ of KIT gene mutations affect exon 11, which codes for the intracellular juxtamembrane domain of the KIT receptor (4). The type of mutations in KIT exon 11 are various, can be either point mutations, deletions, or insertions. Mutations of KIT gene that cause constitutive activation of the tyrosine kinase function of c-kit are detectable in most GISTs and appear to play an important role in tumorigenesis for GIST $(5,6)$.

Mutation screening for GISTs is crucial and has diagnostic value. Besides, the mutation screening offers considerable therapeutic value for guiding the selection of drug, determining medication time and dosage. It has been clinically demonstrated that the sensitivity to targeted therapy and biological behavior of GISTs are associated with the region and type of genetic mutations $(7,8)$. GIST with the primary KIT exon 11 mutation exhibits the best treatment response to Imatinib (Gleevec, Novartis, Basel, Switzerland) (9). Mutations in exon 17 of KIT and exon 18 of PDGFRA are resistant to Imatinib. Exon 9 mutations of KIT are less sensitive to Imatinib, and likely require higher doses of medicine $(10,11)$.

The prognostic data of GISTs is confounded by sensitivities to targeted therapy. GIST with different types of primary KIT exon 11 mutation exhibits different prognostic value. Despite the lack of a clear association between gene mutation and prognosis, some clinical data has shown that deletions involving codons $557-558$ on KIT exon 11 is associated with aggressive biological behavior, the highest recurrence rate after surgery; however, have good response to imatinib (12). Therefore, longer targeted therapy after surgical resection for these patients should be considered.

In general, gene mutations in GISTs are analyzed by examining surgically resected tissue samples. However, a large proportion of GIST patients have no opportunity to receive surgical resection due to metastasis or an enormous size of tumor at the time of diagnosis. Fine-needle aspiration can provide tissue for pathological examination in most cases, however the acquired sample is usually too small for genotyping. Also, owing to its high cost, routine genotype examination is not widely performed, even for surgical resection patients.

Medical imaging is one of the key factors informing medical science. Its superiority lies in its ability to noninvasively assess the characteristics of human tissue and organs. Thus, it is routinely used in clinical practice for oncologic diagnosis as well as treatment guidance and monitoring (13). Computed tomography (CT) is the recommended imaging method for GIST $(14,15)$. Recent advances in medical image acquisition and analysis have allowed for high quality images with isotropy to be obtained. These images contain not only general morphological information, but also rich data. Radiomics has been recognized as an important imaging technology in oncology. It can convert CT images into highthroughput quantitative data, which can reflect intratumor heterogeneity and be associated with gene expression profiles (known as radiogenomics). Radiogenomics is a promising new paradigm for extending clinical imaging into the era of molecular and genomic imaging (16). Recently, several studies demonstrated associations between tumor radiomics features and a certain gene of renal cell carcinoma, breast cancer, and neck tumors $(13,17,18)$.

This study was designed to explore associations between GIST with KIT exon 11 mutation and contrast-enhanced CT images.

We present the following article in accordance with the STARD reporting checklist (available at https://dx.doi. org/10.21037/atm-21-3811).

\section{Methods}

\section{Study population}

This retrospective study was approved by the institutional review board at West China Hospital, Sichuan University (No. 2020-249), and the requirement for written informed consent was waived. All procedures performed in this study involving human participants were in accordance with the 


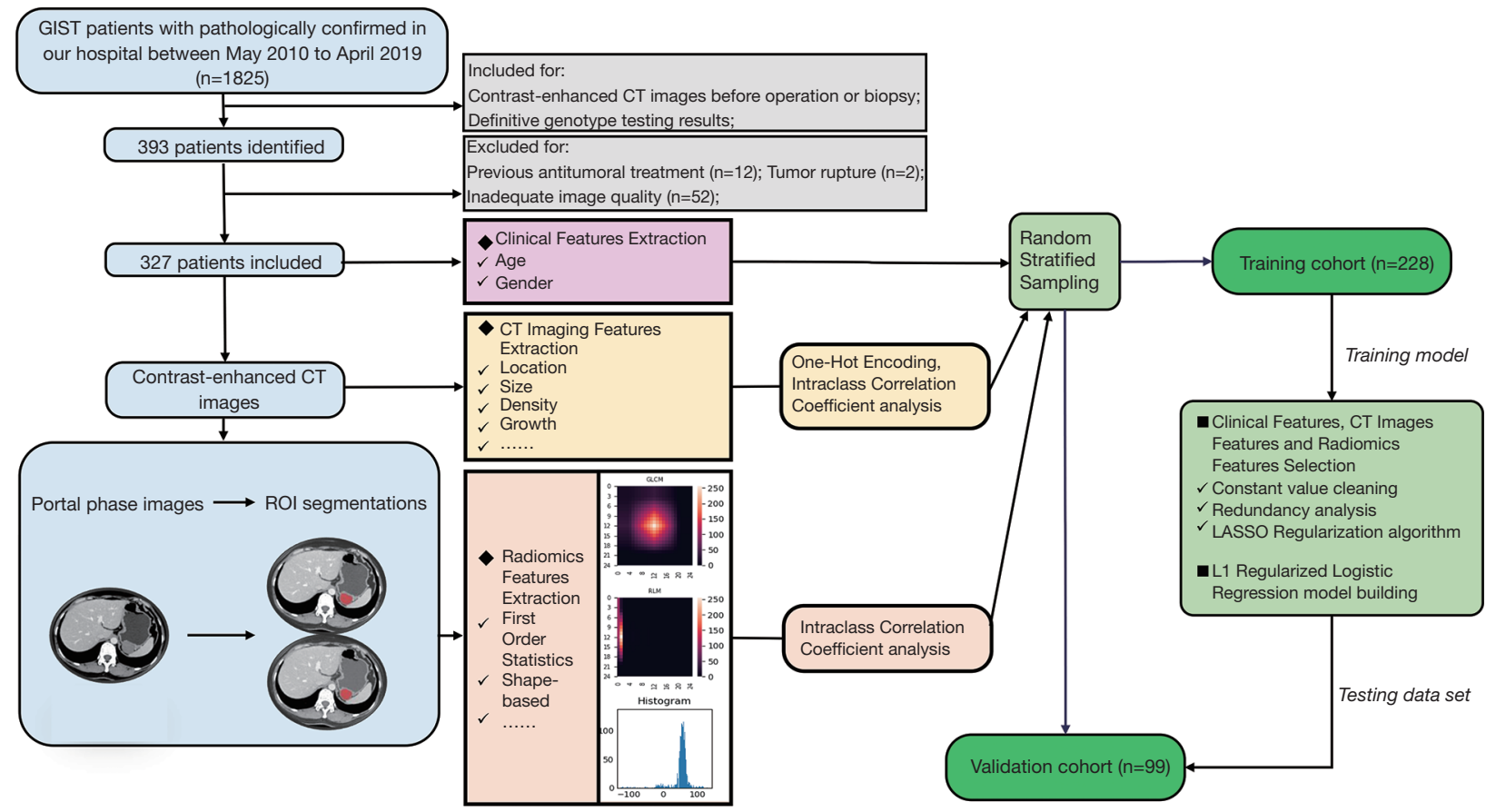

Figure 1 Study workflow. GIST, gastrointestinal stromal tumor; CT, computed tomography; ROI, region of interest; LASSO, least absolute shrinkage and selection operator.

Declaration of Helsinki (as revised in 2013). Pathologically proven GISTs between May 2010 and April 2019 in our hospital were included. The inclusion criteria were as follows: (I) patients who received abdominal contrastenhanced CT images in our hospital prior to surgery or biopsy; and (II) cases with definitive genotype testing results. The exclusion criteria were as follows: (I) those with a previous history of antitumoral treatment (e.g., TKIs therapy, surgery); (II) cases involving inadequate image quality (e.g., severe motion artifact, thickness of portal phase image $>5 \mathrm{~mm}$ ); (III) cases with a time interval between imaging examination and surgery or biopsy of more than 20 days; and (IV) cases involving tumor rupture. In total, 327 patients with 327 GISTs were finally enrolled in this study (Figure 1).

\section{CT protocol}

Bowel cleansing is a precondition for CT examination, which includes a low-residue diet or ample fluids the day before the examination and fasting $10 \mathrm{~h}$ prior to examination. The patients were asked to drink $600-1,000 \mathrm{~mL}$ of water within 40-60 min before the examination.

Among the patients, 123 were examined using a
Brilliance 64 (Philips Medical System, Eindhoven, the Netherlands), 88 were examined using a 128 -slice scanner (SOMATOM Definition AS+, Siemens Healthcare, Germany), and 116 were examined using a dual-source CT system (Somatom Definition Flash, Siemens Healthcare, Germany). The scan range covered the entire abdomen. The scanning parameters were as follows: tube voltage, $120 \mathrm{kV}$; tube current, 145-200 mAs; slice thickness, 2-5 $\mathrm{mm}$; slice interval, $2 \mathrm{~mm}$; field of view, $35-50 \mathrm{~cm}$; matrix, $512 \times 512$; rotation time, $0.5 \mathrm{~s}$; and pitch, 1.0. With a trigger threshold of the aorta reaching 170 Hounsfield unit (HU), enhanced images were obtained with the arterial phase at the trigger and the portal vein phase at $30 \mathrm{~s}$ after the trigger. Iodinated contrast agent $(1.2-1.5 \mathrm{~mL} / \mathrm{kg}$, Iopamiro, Bracco, Italy; Ultravist, Bayer, Germany) was injected intravenously at a flow rate of $2.5-3.0 \mathrm{~mL} / \mathrm{s}$ using a high-pressure syringe (Medrad Stellant CT Injector System, Medrad Inc., Germany).

\section{CT features analyses}

Two radiologists (Xijiao Liu and Caiwei Yang, with 10 and 5 years of experience in abdominal imaging, respectively) independently read the images on Syngo Imaging 
Workplaces (VersionVB35A, Siemens AG, Erlangen, Germany), and were blinded to the clinical and pathological data. Disagreements were resolved by consensus.

CT images features were recorded, including location, size, margin, shape, growth pattern, density, enhancement pattern, enhancement degree, necrosis, calcification, superficial ulceration, gas, cystic change, enlarged vessels feeding or draining the mass (EVFDM), adjacent mesangial fat infiltration, adjacent organ invasion, distal metastasis, and lymphadenopathy. Growth patterns were classified as exophytic, endophytic, and mixed (19). Density was classified as hypodensity, isodensity, or hyperdensity, as compared to paraspinal muscle on unenhanced CT images. Enhancement degree was classified as mild, moderate, and marked (20). Necrosis was an irregular low attenuation region on contrast-enhanced CT scans without obvious enhancement among three phases ( $<10 \mathrm{HU}$ difference) and a CT attenuation value $<20 \mathrm{HU}$ in each phase. A region with smooth and clear border and near-water density (CT attenuation value $0-10 \mathrm{HU}$ ) was defined as cystic degeneration (21). The presence of EVFDM was assessed as previously described (22).

The tumor densities on plain, arterial, and portal venous phases were measured. The regions of interest (ROIs) were defined with $50 \mathrm{~mm}^{2}$, avoiding vessel, gas, and necrosis. The final value was the average of two radiologists.

\section{Radiomics analyses}

For radiomic analysis, ROIs were drawn for each tumor, using ITK-SNAP software (version 3.6.0, http://www.itksnap.org) with hidden patient information. All slices on portal vein phase images were reviewed. The largest crosssection was selected as R1 and the largest cross-section and a nearby cross-section of the tumor were selected as R2 (23). ROIs were required to avoid gas and lumen and were tracked manually (by Xijiao Liu) along $1 \mathrm{~mm}$ from the margin of the tumor to avoid the interference by adjacent tissue. Xijiao Liu and Caiwei Yang repeated the same procedure for of 50 tumors segmentation 2 weeks later to calculate the intraclass correlation coefficients (ICCs).

All ROIs and portal vein serial images were imported using Intelligence Foundry (Version 1.2, General Electric) to extract the radiomics features. Discretization by image filter processing was used to effectively eliminate Gaussian noise. The radiomics features were based on the public package of PyRadiomics (https://pyradiomics.readthedocs. io/en/latest/). Finally, 1,024 radiomics features were extracted (reported in the https://cdn.amegroups.cn/ static/public/atm-21-3811-1.xlsx), including the following categories: Original, Co-occurrence of Local Anisotropic Gradient Orientations (CoLIAGe), and wavelet and local binary pattern feature (Wavelet-LBP). The software automatically extracted the radiomics features while completing the discretization step.

The ICCs of radiomic features extracted from the two sets of ROIs of the 50 tumors (by Xijiao Liu) were calculated for intra-individual comparison. The ICCs of radiomics features extracted from the two sets of ROIs of the 50 tumors (by Xijiao Liu and Caiwei Yang) were also calculated for interindividual comparison. Radiomics features with ICC values greater than 0.75 in intra- and inter-individual comparison were included for model development.

\section{Development and validation of models}

All patients were randomly divided into a training cohort and a validation cohort (at a ratio of 7:3) using the repeated stratified splitting method to reduce the bias selection of a single validation dataset.

The least absolute shrinkage and selection operator (LASSO) algorithm was performed to select the most predictive $\mathrm{CT}$ and radiomics features from the primary dataset. A CT features and radiomics score was calculated using a linear combination of the selected CT features and radiomics features weighted by their LASSO coefficients as: Rad-score $=a_{1} X_{1}+a_{2} X_{2}+\ldots+a_{n} X_{n}+b$. Since the features did not satisfy the normality, we used Spearman's rank correlation coefficient redundancy analysis. The Spearman correlation coefficient showed a value of 0.9 ; that is, for all features, a two-two-correlation calculation was performed. When the coefficient $r \geq 0.9$, the system would randomly delete one feature and retain another feature.

The scores of CT, radiomics, and clinical features (age, gender, and location) were respectively calculated in the training cohort to formulate three nomograms for GISTs with KIT exon 11 mutation and KIT exon 11 deletion involving codons 557-558 using multivariate logistic regression analysis. The performances of the models were evaluated in the validation cohort.

To improve the performance, a five-fold cross-validation of the model was carried out. Numerous features were improved in the regularized L1 logistic regression with penalty term. For better performance of the integrated model, the best $\lambda$ was obtained during the cross-validation procedure. Five independent sub-cohorts were divided 
in the training cohort; four of whom were applied for model fitting, while the remaining sub-group was applied for the validation cohort. Each sub-group was treated as a validation cohort, with the procedure being validated five times. With five times repetition, each sub-group was treated as validation cohort. Finally, $\lambda$ was obtained gained in the cross-validation set, and the results were displayed with regularized L1 logistic regression.

\section{Statistical analyses}

Continuous data were presented as mean \pm standard deviation or median (range), and categorical data were presented as numbers and percentages. Differences in patients with different gene mutation types in both the primary and validation cohorts were assessed using the independent sample t-test or the Mann-Whitney test for continuous variables, and chi-square or Fisher's exact test for categorical variables. The diagnostic performance of the models was evaluated using the receiver operating characteristic (ROC) curve. Delong's test was performed to compare the diagnostic performance of three models.

Inter-observer ICCs were used to measure the interobserver agreement of CT features by Cohen's k statistic. Kappa values greater than 0.75 were considered to represent excellent agreement, values between $0.4-0.75$ signified good agreement, and values below 0.4 denoted poor agreement (24).

Statistical analyses were performed using SPSS software (Version 19, Chicago, IL, USA) and R software (version 1.2.1335, The R Foundation for Statistical Computing, Vienna, Austria). The statistical significance levels were all two-sided, and statistical significance was set at $\mathrm{P}<0.05$.

\section{Results}

\section{Clinicopathological characteristics}

Among the 327 GISTs, 255 tumors were proven with KIT exon 11 mutation and 72 with other types of gene mutation (wild-type mutation, 30; KIT exon 9 mutation, 21; KIT exon 17 mutation, 2; PDGFRA, 14; KIT exon 13 mutation, 5). Among the 255 GISTs with KIT exon 11 mutation, 73 were proven with deletion involving codons 557-558. The median age of patients was 57 years (range, 19-83 years). The demographic and pathological data of the 327 patients are shown in Table 1. There were no significant differences in gender, Ki-67 expression, and risk classification between the KIT exon 11 mutation group and the other types of gene mutation group ( $\mathrm{P}>0.05$ for all). The KIT exon 11 mutation deletion involving codons 557-558 group showed higher Ki-67 expression and a higher risk of aggressive behavior than these without deletion involving codons 557-558.

\section{CT features analyses}

Among the 255 GISTs with KIT exon 11 mutation, there were 193, 58, 2, and 2 tumors located in the stomach, small intestine, rectum, and colon, respectively. Meanwhile, among the 73 GISTs without KIT exon 11 mutation, there were 41,31 , and 1 tumor located in the stomach, small intestine, and rectum, respectively. The locations were statistically different between the two groups $(\mathrm{P}<0.01)$.

The median size of the 327 tumors was $87 \mathrm{~mm}$ (range, 10-320 mm). The tumor size of the KIT exon 11 mutation group was statistically smaller than that without exon 11 mutation [55 mm (range, 11-320 mm) vs. $92 \mathrm{~mm}$ (range, $10-201 \mathrm{~mm}), \mathrm{P}<0.01]$. Irregular shapes were common in both groups. However, irregular shapes of GISTs with KIT exon 11 mutation were significantly less prevalent compared to those without exon 11 mutation $(\mathrm{P}<0.01)$.

The differences in growth patterns were also statistically significant $(\mathrm{P}<0.01)$. The exophytic growth pattern was relatively more common in the GISTs with KIT exon 11 mutation group compared to those without exon 11 mutation $(27.5 \%$ vs. $11.1 \%)$. Moreover, the enhancement degree of the two groups was significantly different $(\mathrm{P}<0.01)$. Twenty-seven (10.6\%) GISTs with exon 11 mutation and $24(33.3 \%)$ GISTs without exon 11 mutation manifested marked enhancement. Both necrosis and superficial ulceration were more common in the GISTs without exon 11 mutation group $(\mathrm{P}<0.01)$.

There were statistical differences in tumor size, margin, shape, growth pattern, enhancement pattern, enhancement degree, necrosis, gas, EVFDM, adjacent mesangial fat infiltration, and adjacent organ invasion between the KIT exon 11 mutation group with deletion involving codons $557-558$ and the other group $(\mathrm{P}<0.01)$.

Details of the CT imaging features are shown in Table 2. Representative CT images indicating the features of KIT exon 11 mutation and KIT exon 11 mutation involving codons 557-558 are shown in Figure 2. 
Table 1 Demographic and pathologic features of included patients

\begin{tabular}{|c|c|c|c|c|c|c|}
\hline & $\begin{array}{c}\text { KIT exon } 11 \\
\text { mutation group }\end{array}$ & $\begin{array}{l}\text { Without KIT exon } \\
11 \text { mutation group }\end{array}$ & $P$ value & $\begin{array}{l}\text { KIT exon } 11 \text { mutation } \\
\text { with deletion involving } \\
\text { codons 557-558 group }\end{array}$ & $\begin{array}{l}\text { KIT exon } 11 \text { mutation } \\
\text { without deletion involving } \\
\text { codons } 557-558 \text { group }\end{array}$ & $P$ value \\
\hline Male & $133(52.1)$ & $41(56.9)$ & 0.473 & $42(57.5)$ & $91(50.0)$ & \\
\hline Female & $122(47.9)$ & $31(43.1)$ & & $31(42.5)$ & $91(50.0)$ & \\
\hline Ki-67 expression & $18(0.5-60)$ & $15(0.5-40)$ & 0.461 & 20 [1-50] & $10[1-60]$ & $<0.01$ \\
\hline \multicolumn{2}{|c|}{ Risk of aggressive behavior*, No. (\%) } & & 0.521 & & & $<0.01$ \\
\hline Very low & $2(0.8)$ & 0 & & 0 & $2(1.1)$ & \\
\hline Low & $55(21.6)$ & $15(20.8)$ & & $5(6.8)$ & $50(27.5)$ & \\
\hline
\end{tabular}

*, according to the modified 2008 National Institute of Health criterion.

\section{Diagnostic performance of models}

A total of 892 radiomics features with ICC values greater than 0.75 in intra- and inter-individual comparison were included for model development. Twelve CT features (including margin, shape, calcification, gas, growth pattern, densities in plain and arterial phase images, cystic change, EVFDM, adjacent mesangial fat infiltration, adjacent organ invasion, and lymphadenopathy), 14 features [including 13 radiomics features and one clinical feature (location)], 14 features including three CT features (gas, growth pattern, and lymphadenopathy), nine radiomics features, and two clinical features (age and location), were finally extracted to build the following models: model ${ }_{[\mathrm{CT}]}, \operatorname{model}_{[\mathrm{radiomic}+\text { clinic] }}$,

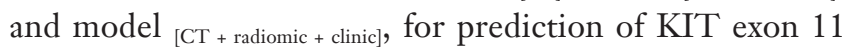
mutation in the training dataset, respectively. The area under curves (AUCs) of the three models were 0.7158 , 0.7530 , and 0.8375 in the training cohort, and 0.6777 , 0.7349 , and 0.8105 in the validation cohort, respectively (Figure $3 A, 3 B$ ). The diagnostic performance of the three models is provided in Table 3. Differences were detected between any paired diagnostic measure for the three models in the training and validation cohorts. The diagnostic accuracy of model [CT + radiomic + clinic] was significantly higher than those of model ${ }_{[\mathrm{CT}]}$ and model $\mathrm{[radiomic}+$ clinic]. $_{\text {. }}$.

Four CT features (size, margin, enhancement degree, and EVFDM), eight features [including seven radiomics features and one clinical feature (location)], 12 features [including three CT features (size, margin, and enhancement degree), eight radiomics features, and one clinical feature (location)] were finally extracted to build the following models: model $_{[\mathrm{CT}]}$, model ${ }_{\text {[radiomic }+ \text { clinic] }}$ and model ${ }_{[\mathrm{CT}+\text { radiomic }+ \text { clinic] }}$, for prediction of KIT exon 11 mutation with deletion involving codons $557-558$ in the training dataset, respectively. The AUCs of the three models were $0.7155,8621$, and 0.8691 in the training cohort, and $0.7099,0.8355$, and 0.8488 in the validation cohort, respectively (Figure $3 C, 3 D$ ). The diagnostic performance of the three models is provided in Table 3. Significant differences were detected between any paired diagnostic measure for the three models in the training and validation cohorts. Model $[\mathrm{CT}+$ radiomic + clinic] demonstrated the highest diagnostic performance.

\section{Interrater agreement assessment}

The kappa value between two radiologists for the qualitative analysis of CT image features was 0.802 , which indicated excellent agreement.

\section{Discussion}

This study aimed to explore the association between GIST with KIT exon 11 mutation and contrast-enhanced CT images derived from large-scale imaging data. Significant differences in CT features were found between GIST with KIT exon 11 mutation group and without KIT exon 11 
Table 2 CT imaging features of included GISTs

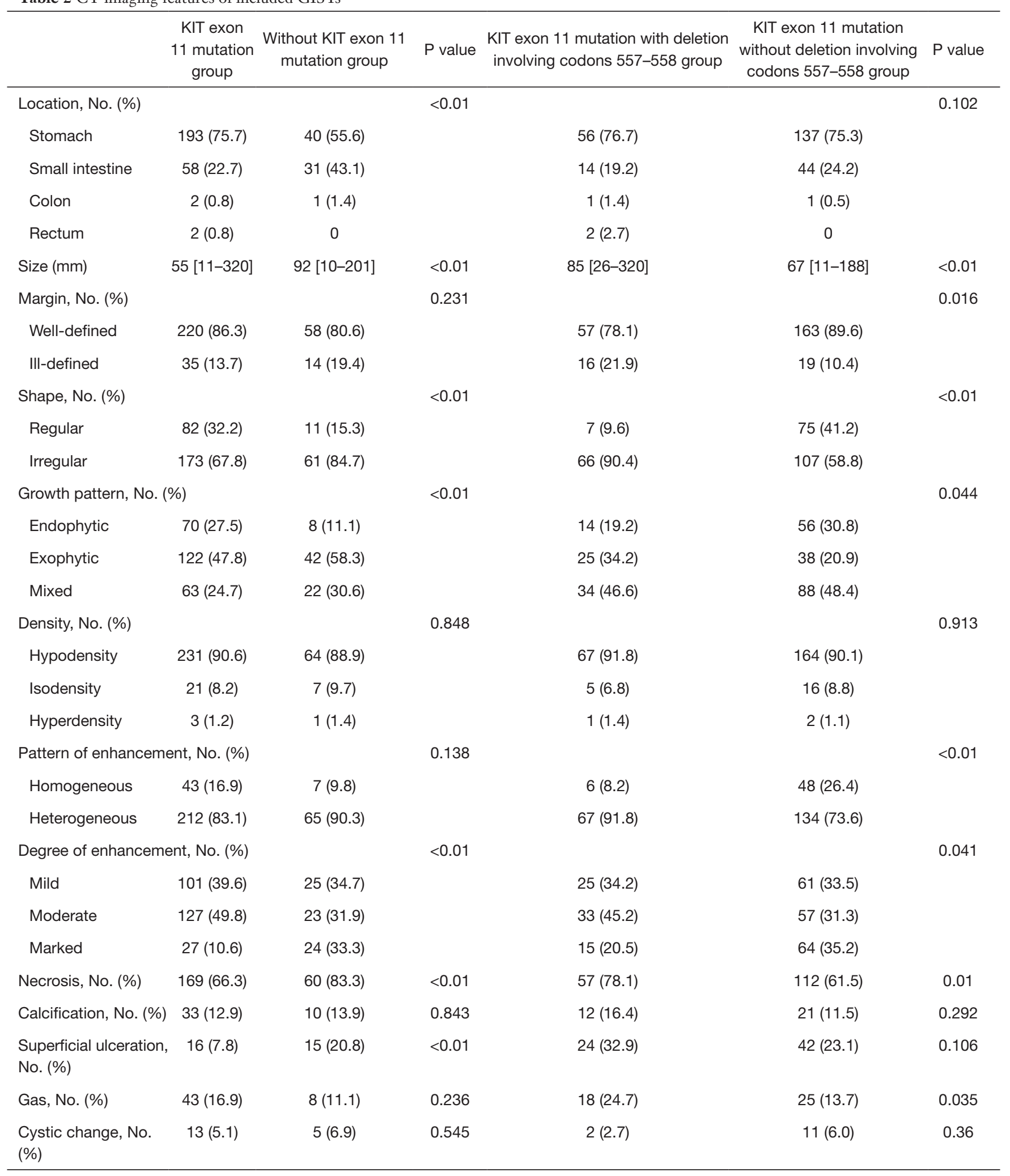

Table 2 (continued) 
Table 2 (continued)

\begin{tabular}{|c|c|c|c|c|c|c|}
\hline & $\begin{array}{l}\text { KIT exon } \\
11 \text { mutation } \\
\text { group }\end{array}$ & $\begin{array}{l}\text { Without KIT exon } 11 \\
\text { mutation group }\end{array}$ & $P$ value & $\begin{array}{l}\text { KIT exon } 11 \text { mutation with deletion } \\
\text { involving codons } 557-558 \text { group }\end{array}$ & $\begin{array}{l}\text { KIT exon } 11 \text { mutation } \\
\text { without deletion involving } \\
\text { codons } 557-558 \text { group }\end{array}$ & $P$ value \\
\hline $\begin{array}{l}\text { Adjacent mesangial } \\
\text { fat infiltration, No. (\%) }\end{array}$ & $63(24.7)$ & $20(27.8)$ & 0.597 & 27 (37.0) & 36 (19.8) & $<0.01$ \\
\hline $\begin{array}{l}\text { Distal metastasis, } \\
\text { No. }(\%)\end{array}$ & $6(2.4)$ & $5(6.9)$ & 0.057 & $4(5.5)$ & $2(1.1)$ & 0.058 \\
\hline $\begin{array}{l}\text { Lymphadenopathy, } \\
\text { No. (\%) }\end{array}$ & $21(8.2)$ & $7(9.7)$ & 0.691 & $8(11.0)$ & $13(7.1)$ & 0.316 \\
\hline
\end{tabular}

EVFDM, enlarged vessels feeding or draining the mass.
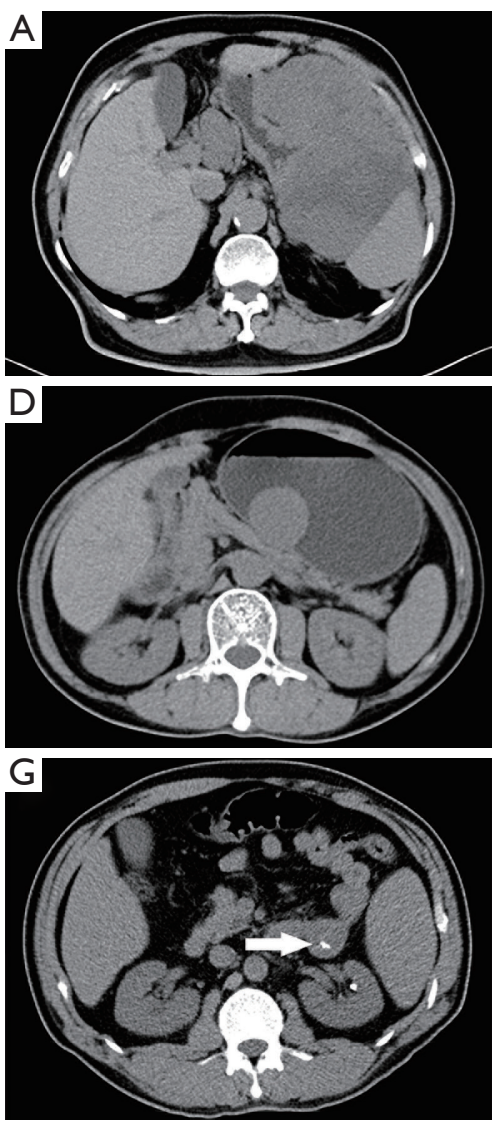
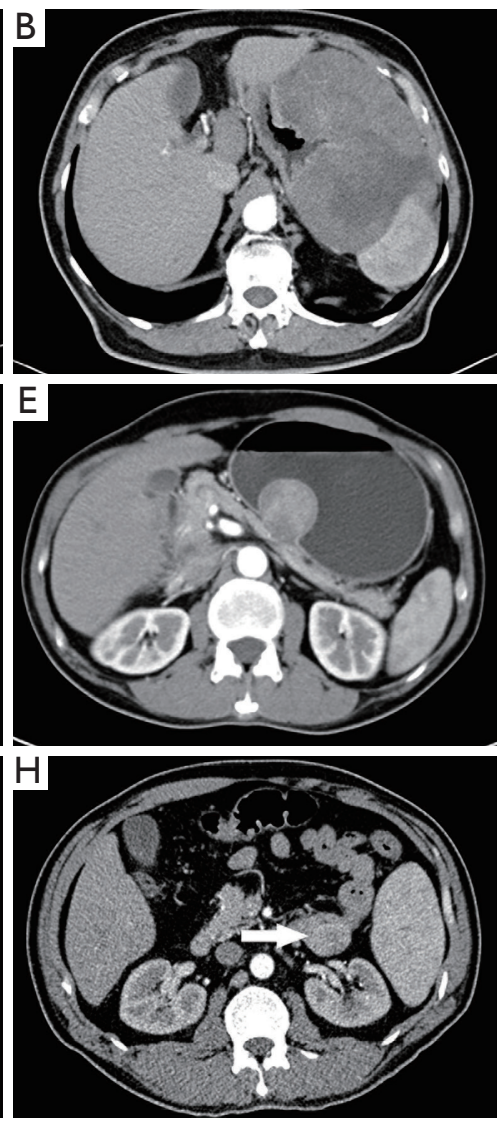
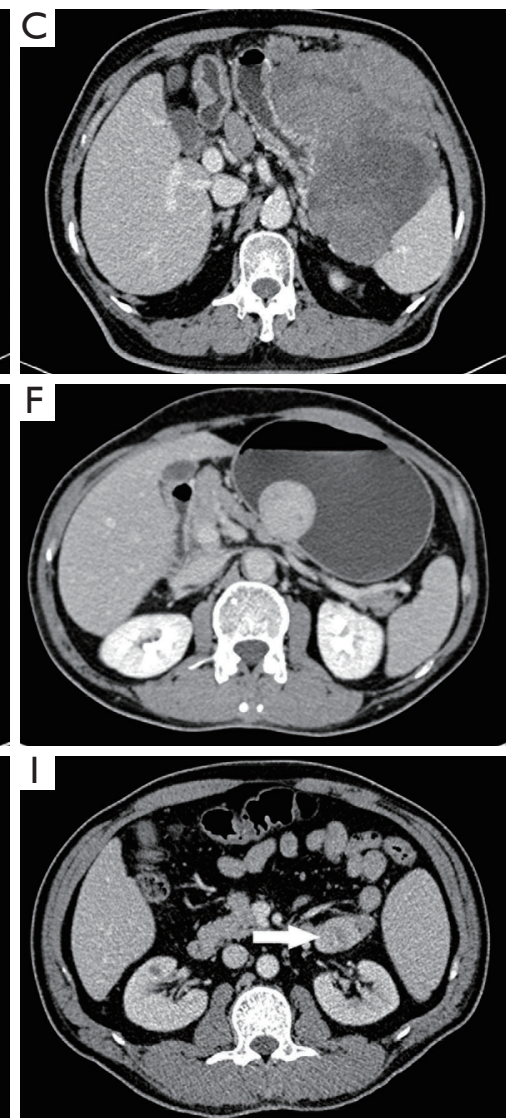

Figure 2 Gastrointestinal stromal tumor (GIST) in the stomach with KIT exon 11 mutation deletion involving codons 557-558 in an 82-year-old male (A, plain CT image; B, artery phase image; C, portal phase image). GIST in the stomach with KIT exon 11 mutation with non-deletion involving codons 557-558 in a 61-year-old male (D, plain CT image; E, artery phase image; F, portal phase image). GIST in the small intestine with non-KIT exon 11 mutation in a 41-year-old male (G, plain CT image; H, artery phase image; I, portal phase image; arrow, the tumor). 

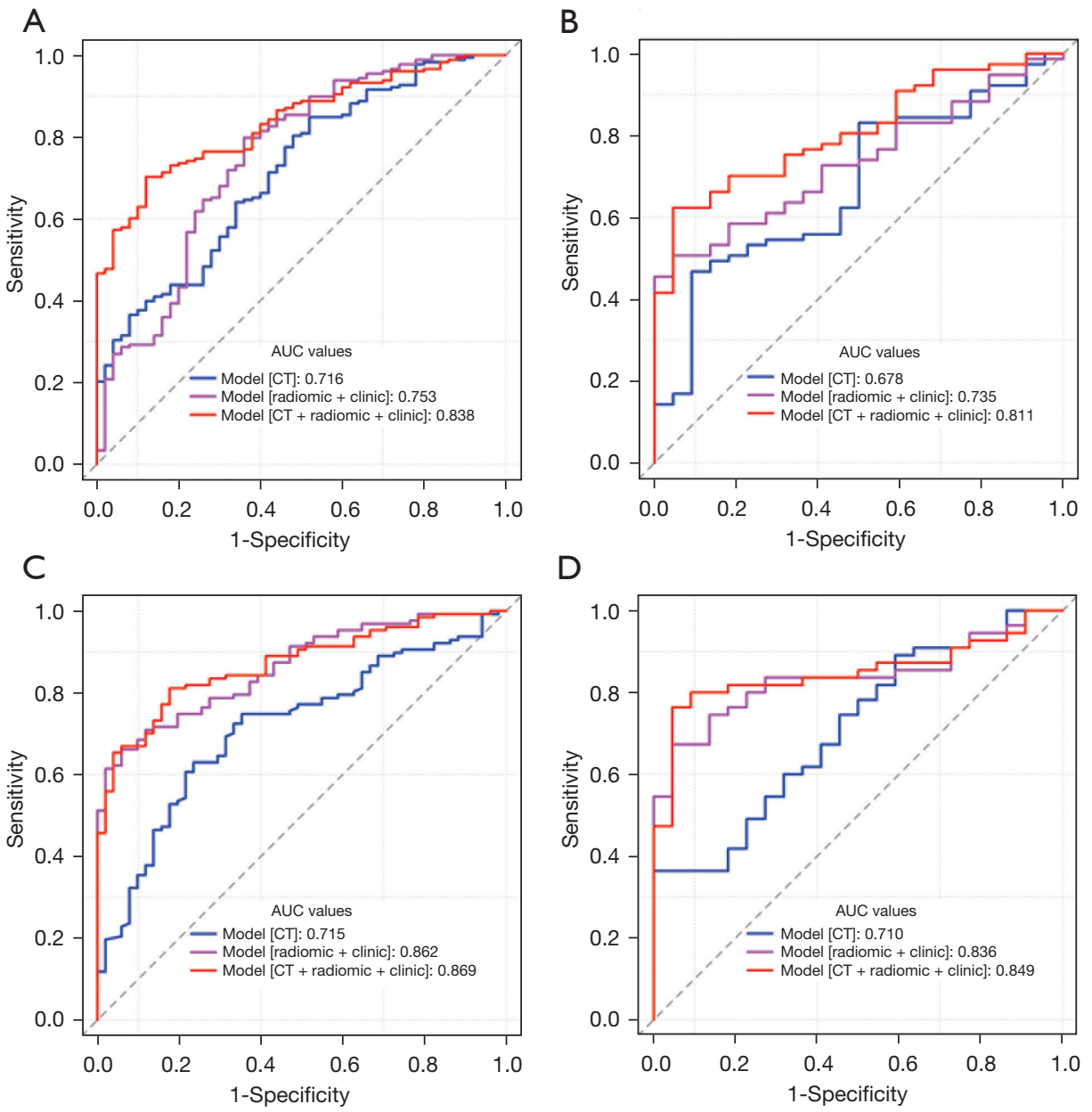

Figure 3 The area under curves (AUCs) of model (CT), model (radiomic + clinic), and model (cT + radiomic + clinic $)$ for prediction of KIT exon 11 mutation and KIT exon 11 mutation with deletion involving codons 557-558. The AUCs of three models for prediction of KIT exon 11 mutation in the training data (A) and the validation cohort (B). The AUCs of three models for prediction of KIT exon 11 mutation with deletion involving codons 557-558 in the training data (C) and the validation cohort (D).

mutation group, as well as between GIST with KIT exon 11 mutation group with deletion involving codons 557-558 and without deletion involving codons 557-558 group. Model ${ }_{[\mathrm{CT}+\text { radiomic }+ \text { clinic] }}$ demonstrated the highest diagnostic performance for prediction of KIT exon 11 mutation and those with deletion involving codons 557-558, which meant that the judgment efficiency of combing conventional image analysis and texture analysis is better than the conventional image analysis or texture analysis alone. The model ${ }_{\text {[radiomic + clinic] }}$ demonstrated higher diagnostic performance than model ${ }_{[\mathrm{CT}]}$ significantly. Hence, we believe CT texture analysis is a useful tool to distinguish GIST with KIT exon 11 mutations and the best tool is combing conventional image analysis and texture analysis.
In our study, GISTs with KIT exon 11 mutation were mainly located in the stomach, which is consistent with previous reports. Compared to GIST with other types of mutations, GISTs with KIT exon 11 mutation typically exhibit the following characteristics: small tumor size, welldefined, exophytic growth pattern, and hypodensity on plain CT image. Irregular shape, necrosis, and EVFDM could also be observed. These GISTs always manifest heterogeneous enhancement and a mild to moderated degree of enhancement. Superficial ulceration is an extremely rare feature, while cystic change, distal metastasis, and lymphadenopathy features are seldom occurrences.

Previous studies have reported that KIT exon 11 mutation with deletion involving codons 557-558 may 


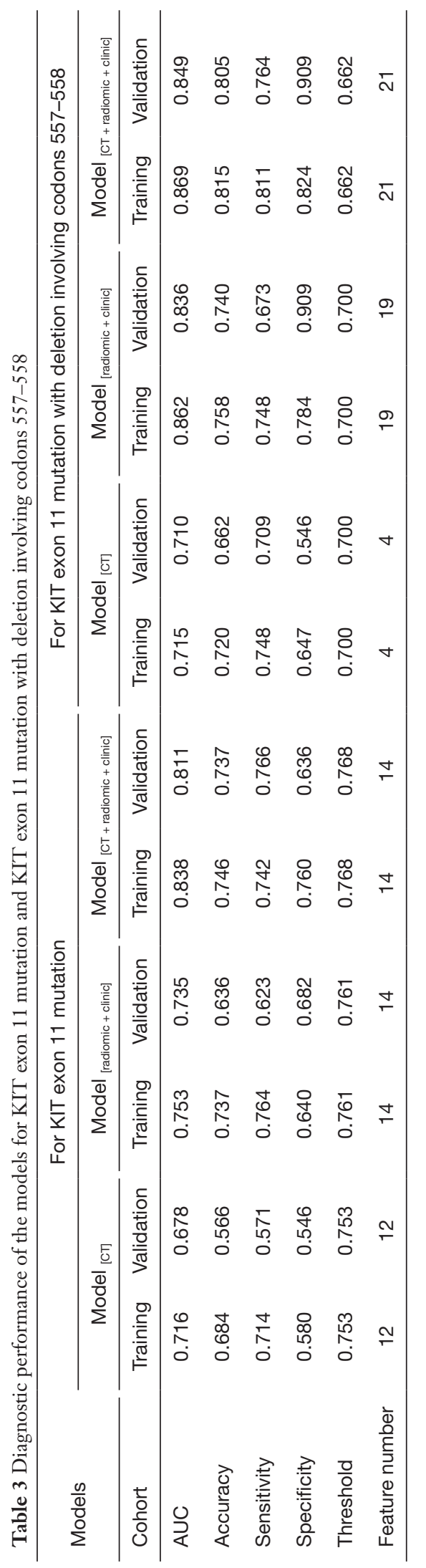

be associated with aggressive biological behavior (5). Our results showed KIT exon 11 mutation deletion involving codons 557-558 had a higher Ki-67 expression and a higher risk of aggressive behavior than other types of exon 11 mutations. On CT images, GISTs with KIT exon 11 mutation with deletion involving codons 557-558 were tumors with a relatively larger size and exophytic and mixed growth pattern. Irregular shape, heterogeneous enhancement. And EVFDM were significantly more common on CT images. Mild and moderate degrees of enhancement, and necrosis were common CT features. Although infrequent in GISTs with KIT exon 11 mutation, our study found that the manifestations of features, including gas, adjacent mesangial fat infiltration, and adjacent organ invasion, were indications for mutation with deletion involving codons 557-558.

Traditionally, medical imaging has been a subjective or qualitative science. Radiomics could convert these images into high-throughput quantitative data, which could reflect intra-tumor heterogeneity and be associated with gene expression profiles called radiogenomics. In 2018, Xu et al. first reported that CT texture analysis could potentially help to differentiate GISTs without KIT exon 11 mutation from those with KIT exon 11 mutation on enhanced CT images. They observed that the standard deviation of texture parameters of tumors without KIT exon 11 mutation is an independent predictor of no KIT exon 11 mutation. However, the sample sizes of the training and validation sets were 69 and 17 cases, respectively, and there were only 4 cases of tumors without KIT exon 11 mutation in the validation set, which may have affected the accuracy of the results. In addition, only 30 radiomics features were extracted from portal phase CT images for texture analysis in their study. Finally, it is generally recognized that targeted therapy may alter the imaging manifestation of GISTs and induce secondary gene mutation. In their study, authors did not mention this factor.

Compared to the study by $\mathrm{Xu}$ et al., our study makes up for the above deficiencies and demonstrates progress. Firstly, our study included a relatively larger cohort. Secondly, we analyzed 1,024 radiomics features for radiogenomics, and traditional CT image features, such as morphologic and density parameters, were also analyzed. Thirdly, we applied definite exclusion criteria; GISTs with previous history of antitumoral treatment (e.g., TKI therapy, surgery) were excluded.

The statistical method used in our study has several strengths. Firstly, in order to reduce the differences 
between the training and verification sets and improve the stability of the two, we chose random stratified sampling for data segmentation rather than random allocation. This ensured that the distribution of the training and validation sets at the gold standard level (i.e., the proportion of total mutations) was consistent. Secondly, we took all clinical, $\mathrm{CT}$, and radiomic features into account in the variable analysis, and one-hot encoding of features of categories data was performed. In this way, the validity of features could be analyzed and the efficiency of the model could be improved. Thirdly, the LASSO algorithm was adapted to efficiently reduce dimension in terms of feature screening level. In the model building, we added L1 regularization to the logistic regression, which enhanced model stability and affinity to the real function.

However, the present study has several limitations that should be noted. Firstly, the retrospective design and exclusion criterion of patients could lead to a certain selection bias. Secondly, due to the small sample size, we grouped GISTs with gene mutations with no KIT exon 11 mutation together. It is important to differentiate certain types of mutations, such as small intestinal GIST with KIT exon 11 mutation and that with KIT exon 9 mutation. This should be considered in future studies with a large sample size or a specific design. Thirdly, we investigated the association between GISTs with KIT exon 11 mutation and contrast-enhanced CT images and the results demonstrated a relationship between them. Unfortunately, we did not perform further research on the molecular and pathological factors that lead to this correlation. Fourthly, CT examinations were performed on three different scanners. However, we assumed that this would give our models reproducibility, robustness, and potentially enable their wide diffusion. Lastly, although our research was based on large-scale data, it was a single-center study, which may weaken the generalizability of the results. Thus, further validation from multi-center populations is required.

Our study presented evidence for contrast-enhanced CT images of GIST with KIT exon 11 mutation as well as GIST with KIT exon 11 mutation with deletion involving codons 557-558. CT radiogenomics shows good application in predicting these tumors. Owing to the automated feature algorithms, the results of this study could stimulate further research of image-based quantitative features. This approach could have a considerable impact, as CT imaging is routinely used worldwide in all stages of GIST diagnoses and treatment, and provide a non-invasive opportunity to predict gene mutation types.

\section{Acknowledgments}

Funding: This work was supported by the National Nature Science Foundations of China (grant number: 82001810) and the 1.3.5 project for disciplines of Excellence-Clinical Research Incubation Project, West China Hospital, Sichuan University (grant number: 19HXFH054).

\section{Footnote}

Reporting Checklist: The authors have completed the STARD reporting checklist. Available at https://dx.doi. org/10.21037/atm-21-3811

Data Sharing Statement: Available at https://dx.doi. org/10.21037/atm-21-3811

Conflicts of Interest: All authors have completed the ICMJE uniform disclosure form (available at https://dx.doi. org/10.21037/atm-21-3811). Dr. BS serves as an unpaid editorial board member of Annals of Translational Medicine from Sept 2021 to Aug 2022. The other authors have no conflicts of interest to declare.

Ethical Statement: The authors are accountable for all aspects of the work in ensuring that questions related to the accuracy or integrity of any part of the work are appropriately investigated and resolved. This retrospective study was approved by the institutional review board at West China Hospital, Sichuan University (No. 2020-249), and the requirement for written informed consent was waived. All procedures performed in this study involving human participants were in accordance with the Declaration of Helsinki (as revised in 2013).

Open Access Statement: This is an Open Access article distributed in accordance with the Creative Commons Attribution-NonCommercial-NoDerivs 4.0 International License (CC BY-NC-ND 4.0), which permits the noncommercial replication and distribution of the article with the strict proviso that no changes or edits are made and the original work is properly cited (including links to both the formal publication through the relevant DOI and the license). See: https://creativecommons.org/licenses/by-nc-nd/4.0/.

\section{References}

1. Ma GL, Murphy JD, Martinez ME, et al. Epidemiology 
of gastrointestinal stromal tumors in the era of histology codes: results of a population-based study. Cancer Epidemiol Biomarkers Prev 2015;24:298-302.

2. Yang Z, Guo W, Huang R, et al. Transanal versus nontransanal surgery for the treatment of primary rectal gastrointestinal stromal tumors: a 10-year experience in a high-volume center. Ann Transl Med 2020;8:201.

3. Heinrich MC, Corless CL, Duensing A, et al. PDGFRA activating mutations in gastrointestinal stromal tumors. Science 2003;299:708-10.

4. Corless CL, Barnett CM, Heinrich MC. Gastrointestinal stromal tumours: origin and molecular oncology. Nat Rev Cancer 2011;11:865-78.

5. Martín J, Poveda A, Llombart-Bosch A, et al. Deletions affecting codons 557-558 of the c-KIT gene indicate a poor prognosis in patients with completely resected gastrointestinal stromal tumors: a study by the Spanish Group for Sarcoma Research (GEIS). J Clin Oncol 2005;23:6190-8.

6. Martin-Broto J, Gutierrez A, Garcia-Del-Muro X, et al. Prognostic time dependence of deletions affecting codons 557 and/or 558 of KIT gene for relapse-free survival (RFS) in localized GIST: a Spanish Group for Sarcoma Research (GEIS) Study. Ann Oncol 2010;21:1552-7.

7. Wang $\mathrm{M}, \mathrm{Xu} \mathrm{J}$, Zhao $\mathrm{W}$, et al. Prognostic value of mutational characteristics in gastrointestinal stromal tumors: a singlecenter experience in 275 cases. Med Oncol 2014;31:819.

8. Ramaswamy A, Bal M, Swami R, et al. Early outcomes of exon 11 mutants in GIST treated with standard dose Imatinib. Ann Transl Med 2017;5:134.

9. Demetri GD, von Mehren M, Blanke CD, et al. Efficacy and safety of imatinib mesylate in advanced gastrointestinal stromal tumors. N Engl J Med 2002;347:472-80.

10. Verweij J, Casali PG, Zalcberg J, et al. Progression-free survival in gastrointestinal stromal tumours with high-dose imatinib: randomised trial. Lancet 2004;364:1127-34.

11. Miettinen M, Sobin LH, Lasota J. Gastrointestinal stromal tumors of the stomach: a clinicopathologic, immunohistochemical, and molecular genetic study of 1765 cases with long-term follow-up. Am J Surg Pathol 2005;29:52-68.

12. Bachet JB, Hostein I, Le Cesne A, et al. Prognosis and predictive value of KIT exon 11 deletion in GISTs. Br J Cancer 2009;101:7-11.

13. Aerts HJ, Velazquez ER, Leijenaar RT, et al. Decoding tumour phenotype by noninvasive imaging using a quantitative radiomics approach. Nat Commun 2014;5:4006.
14. Judson I, Bulusu R, Seddon B, et al. UK clinical practice guidelines for the management of gastrointestinal stromal tumours (GIST). Clin Sarcoma Res 2017;7:6.

15. Xu D, Si GY, He QZ. Correlation analysis of multislice computed tomography (MSCT) findings, clinicopathological factors, and prognosis of gastric gastrointestinal stromal tumors. Transl Cancer Res 2020;9:1787-94.

16. Kuo MD, Jamshidi N. Behind the numbers: Decoding molecular phenotypes with radiogenomics--guiding principles and technical considerations. Radiology 2014;270:320-5.

17. Grimm LJ, Zhang J, Mazurowski MA. Computational approach to radiogenomics of breast cancer: Luminal A and luminal B molecular subtypes are associated with imaging features on routine breast MRI extracted using computer vision algorithms. J Magn Reson Imaging 2015;42:902-7.

18. Karlo CA, Di Paolo PL, Chaim J, et al. Radiogenomics of clear cell renal cell carcinoma: associations between CT imaging features and mutations. Radiology 2014;270:464-71.

19. Kim HC, Lee JM, Kim KW, et al. Gastrointestinal stromal tumors of the stomach: CT findings and prediction of malignancy. AJR Am J Roentgenol 2004;183:893-8.

20. Zhou C, Duan X, Zhang X, et al. Predictive features of CT for risk stratifications in patients with primary gastrointestinal stromal tumour. Eur Radiol 2016;26:3086-93.

21. Su Q, Wang Q, Zhang H, et al. Computed tomography findings of small bowel gastrointestinal stromal tumors with different histologic risks of progression. Abdom Radiol (NY) 2018;43:2651-8.

22. Yin YQ, Liu CJ, Zhang B, et al. Association between CT imaging features and KIT mutations in small intestinal gastrointestinal stromal tumors. Sci Rep 2019;9:7257.

23. Xu F, Ma X, Wang Y, et al. CT texture analysis can be a potential tool to differentiate gastrointestinal stromal tumors without KIT exon 11 mutation. Eur J Radiol 2018;107:90-7.

24. Landis JR, Koch GG. The measurement of observer agreement for categorical data. Biometrics 1977;33:159-74.

(English Language Editor: A. Kassem)

Cite this article as: Liu $\mathrm{X}$, Yin $\mathrm{Y}$, Wang $\mathrm{X}$, Yang $\mathrm{C}$, Wan S, Yin X, Wu T, Chen H, Xu Z, Li X, Song B, Zhang B. Gastrointestinal stromal tumors: associations between contrastenhanced CT images and KIT exon 11 gene mutation. Ann Transl Med 2021;9(19):1496. doi: 10.21037/atm-21-3811 